\title{
The role of mechanical signals in regulating chondrogenesis and osteogenesis of mesenchymal stem cells
}

\author{
Kelly, D.J. ${ }^{1}$ Jacobs, C.R. ${ }^{2}$ \\ ${ }^{1}$ Trinity Centre for Bioengineering, School of Engineering, Trinity College Dublin, \\ Ireland. \\ ${ }^{2}$ Department of Biomedical Engineering, Columbia University, New York, USA.
}

Keywords: Mesenchymal Stem Cell, Osteogenesis, Chondrogenesis, Mechanobiology, Mechano-transduction, Bioreactor. 


\begin{abstract}
It is becoming increasingly clear that Mesenchymal Stem Cell (MSC) differentiation is regulated by mechanical signals. Mechanical forces generated intrinsically within the cell in response to its extracellular environment, and extrinsic mechanical signals imposed upon the cell by the extracellular environment, play a central role in determining MSC fate. This paper reviews chondrogenesis and osteogenesis during skeletogenesis, and then considers the role of mechanics in regulating limb development and regenerative events such as fracture repair. However, observing skeletal changes under altered loading conditions can only partially explain the role of mechanics in controlling MSC differentiation. Increasingly, understanding how epigenetic factors such as the mechanical environment regulate stem cell fate is undertaken using tightly controlled in vitro models. Factors such as bio-engineered surfaces, substrates and bioreactor systems are used to control the mechanical forces imposed upon, and generated within, MSCs. From these studies, a clearer picture of how osteogenesis and chondrogenesis of MSCs is regulated by mechanical signals is beginning to emerge. Understanding the response of MSCs to such regulatory factors is a key step towards understanding their role in disease and regeneration.
\end{abstract}




\section{Introduction}

There is abundant evidence in the literature, from studies of embryonic limb development to investigations of adult skeletal tissue damage and repair that mechanical signals in some way regulate chondrogenesis and osteogenesis of mesenchymal stem cells (MSCs). While advances in molecular biology have allowed a systematic investigation of the role of various genes in regulating the differentiation and growth of skeletal cells and tissues, the role of epigenetic factors such as mechanical signals are less well understood. We are only beginning to understand how stem cells sense their mechanical environment, how these signals are translated into a cascade of biochemical events within the cell and what changes in gene expression and differentiation result from these external signals. Understanding these processes is complex, as it is becoming increasing clear that the response of MSCs to extrinsic mechanical signals depends on the state of the cytoskeletal network, and correspondingly, the dynamics of the cytoskeletal network are regulated by mechanical signals generated outside the cell. This paper will review recent in vivo, in vitro and computational studies that are starting to describe how mechanical signals can act alone or in concert with other regulatory factors such as soluble mediators to control chondrogenesis and osteogenesis of stem cells and their progeny.

\section{Osteoblast and Chondrocyte Differentiation during Skeletogenesis}

There are two mechanisms through which bone formation occurs, intramembranous and endochondral ossification. Both processes begin with stem cell proliferation and condensation (Karsenty and Wagner 2002; Goldring et al., 2006). During intramembranous ossification, these condensed MSCs differentiate directly into osteoblasts. During development, this process occurs mainly in the head region. The development of long bones, on the other hand, takes place by endochondral ossification, where the MSCs first differentiate into proliferating chondrocytes and form the initial cartilage templates. Chondrocytes in the centre of these developing cartilage elements further differentiate into hypertrophic chondrocytes, while stem cells in the perichondrium (the peripheral tissue of the developing limb) differentiate into osteoblasts and form a collar of bone around the hypertrophic chondrocytes. Next, the hypertrophic cartilage is invaded by blood vessels and eventually replaced by bone and marrow. In humans and other mammals, secondary centres of 
ossification form at the distal ends of the developing limbs, leaving a growth plate between the ossification centres which provides the mechanism for further postnatal growth.

Advances in molecular biology have provided significant insight into the genetic mechanisms regulating chondrocyte and osteoblast differentiation during skeletogenesis. Two transcription factors, Sox9 and Runx2, have been demonstrated to be important in the differentiation pathways of these cell types. The transcription factor Sox9 acts during early chondrogenic differentiation. If Sox9 is inactivated in the mouse, the mesenchyme does not express chondrocyte-specific markers such as Col2a1 and Aggrecan, and cartilage does not form (Bi et al., 1999). Runx2 is a transcription factor essential for osteoblast differentiation and bone development (Ducy et al., 1997; Komori et al., 1997; Otto et al., 1997). In the early mesenchymal condensations stem cells express both of these transcription factors, indicating the potential to differentiate along either the osteogenic or chondrogenic lineage (Ducy et al., 1997; Otto et al., 1997; Bi et al., 1999; Yamashiro et al., 2004). However, since genetic inactivation of these transcription factors leads to loss of one cell phenotype without commitment to another, it suggests that other factors must act upstream of Runx2 and Sox9 in controlling osteogenesis and chondrogenesis (Day et al., 2005).

Extrinsic environmental factors may regulate stem cell differentiation by controlling the expression of intrinsic factors such as Runx2 and Sox9 in stem cells, with cell-cell and cell-matrix signals in the condensing mesenchyme playing critical roles. There is strong evidence that the expression of many Wnts during skeletal development are key signals in regulating chondrocyte and osteoblast differentiation (Day and Yang 2008). Wnts are a large family of secreted molecules that transduce their signals through a number of different pathways (Gordon and Nusse 2006). Of these, the canonical or Wnt/ $\beta$-catenin signalling pathway has been shown to control osteoblast and chondrocyte differentiation from mesenchymal progenitors (Day et al., 2005; Day and Yang 2008). This pathway controls the protein stability of $\beta$-catenin, a transcription factor in the canonical Wnt pathway that is also involved in cell adhesion by binding to cadherins (Perez-Moreno and Fuchs 2006) (calcium dependant adhesion molecules that play important roles in cell adhesion). In the absence of Wnt ligands, $\beta$-catenin is phosphorylated and tagged for degradation. When Wnt ligands bind to their co-receptors at the cell membrane, it initiates a sequence of events leading to accumulation of $\beta$-catenin in the cytoplasm and translocation to the 
nucleus, where it activates downstream target genes. Canonical Wnt signalling is upregulated in osteogenic mesenchymal condensations (Day et al., 2005). Conditional knock-out of $\beta$-catenin in mouse mesenchymal condensations leads to a loss of osteoblasts and ectopic chondrocyte differentiation in both intramembranous and endochondral ossification (Day et al., 2005). In addition, inactivation of $\beta$-catenin in MSCs in vitro results in chondrogenic differentiation even in osteogenic culture conditions (Day et al., 2005). Taken together, these results suggest that Wnt/ $\beta$-catenin signalling controls osteogenic and chondrogenic differentiation in mesenchymal condensations, see Fig. 1. As will be discussed in Section 5, there is emerging evidence to suggest that this signalling pathway may, at least in part, be regulated by mechanical signals.

\section{Role of Mechanical Signals in Regulating Limb Development and Fracture Repair}

Beginning with the pioneering work of Wilhelm Roux in the late $19^{\text {th }}$ and early $20^{\text {th }}$ century, numerous authors have hypothesized that the mechanical environment in a developing or regenerating limb regulates the differentiation of skeletal tissues. A number of excellent review papers are available in the literature that consider the role of the mechanical environment in embryonic limb development (Estes et al., 2004; Nowlan et al., 2007; Knothe Tate et al., 2008). While the literature describing in vivo and in vitro investigations of the mechanobiology of embryonic limb development is often disjointed and contradictory, a number of important findings clearly establish a central role of mechanical factors. The level of tension experienced by cultured embryonic bone rudiments has been shown to regulate the rate of bone formation (Glucksmann 1939; Glucksmann 1942), while intermittent compression of cultured embryonic mouse long bone rudiments has been shown to increase calcification of the growth plate cartilage (Klein-Nulend et al., 1986). In vitro studies have also demonstrated that cartilaginous tissue in the membrane bones of the embryonic chick will form in response to the mechanical signals generated by movement (Hall 1968). Chondrogenesis of limb bud mesenchymal cells embedded in collagen or agarose hydrogels has also been shown to be enhanced by compressive loading (Takahashi et al., 1998; Elder et al., 2000; Elder et al., 2001).

Immobilization of the developing embryo through the use of neuromuscular agents and other means has also been used to investigate the role of mechanical 
stimulation on limb development. Mobilisation is known to be required for normal joint cavitation (Mikic et al., 2000; Osborne et al., 2002). Paralysis of the skeletal musculature is also known to inhibit chondrogenesis in developing limbs (Murray and Drachman 1969; Hall 1972; Hall 1979). Maintenance of a chondrogenic as apposed to osteoblastic phenotype is also dependent on normal physiological contraction of the adjacent musculature (Petrovic 1974). While paralysis does not prevent progenitor cells from undergoing osteogenesis in developing limbs, it would appear to regulate the length, mass and mechanical properties of many developing bones (Hall and Herring 1990; Hosseini and Hogg 1991; Hosseini and Hogg 1991; Wong et al., 1993). For example, diminished growth of the skeleton has been correlated with reduced muscular activity (Hall and Herring 1990). In addition, when muscle formation is disrupted, skeletal development is differentially affected, with certain rudiments being more severely diminished than others, suggesting a complex interaction between mechanical forces and other location-specific regulatory factors (Nowlan et al., ). The cartilaginous tissue within developing bones is also influenced by immobilization, with lower glycosaminoglycan (GAG) content and weaker mechanical properties reported compared to control embryos (Mikic et al., 2004).

Endochondral ossification also occurs during regenerative events such as bone fracture repair. While many of the genetic mechanism regulating fetal skeletogenesis also play a role in adult skeletal regeneration (Vortkamp et al., 1998; Ferguson et al., 1999), the mechanical environment is known to play a key role in regulating the outcomes of fracture repair (Goodship and Kenwright 1985; Kenwright and Goodship 1989; Kenwright et al., 1991; Chao et al., 1998; Goodship et al., 1998; Goodship et al., 2009). A number of hypotheses have been proposed for how MSC differentiation is regulated by the mechanical environment during events such as fracture repair. Pauwels suggested that stress and strain invariants guide the differentiation pathway, whereby hydrostatic pressure results in cartilage formation; while distortional strain, or elongation, favours fibrous tissue formation (Pauwels 1941; Pauwels 1960). A similar concept has been adopted by other authors to explain a number of mechanobiological processes (Carter et al., 1998; Loboa et al., 2001; Henderson and Carter 2002). Fluid flow and shear strain have also been hypothesized as the stimuli that direct MSC differentiation during skeletal regeneration (Prendergast et al., 1997). Using this mechano-regulation model (Prendergast et al., 1997), it has been possible to predict the spatial and temporal patterns of tissue differentiation during a number of 
regenerative events such as fracture healing (Lacroix and Prendergast 2002; Lacroix and Prendergast 2002; Isaksson et al., 2006; Isaksson et al., 2006; Nagel and Kelly 2009), osteochondral defect repair (Kelly and Prendergast 2005; Kelly and Prendergast 2006) and distraction osteogenesis (Boccaccio et al., 2007; Boccaccio et al., 2008). However even with the use of complex computational models, it is difficult to determine how specific mechanical signals influence MSC differentiation in vivo within the milieu of other regulatory factors that are present. For example, the mechanical environment within a fracture callus could potentially regulate endochondral ossification in a number of ways. Firstly, the biophysical stimuli that act on the MSCs within the defect could directly promote differentiation along the chondrogenic pathway. Secondly, by preventing neo-vascularization and promoting a local hypoxic environment, mechanical loading could indirectly promote chondrogenesis of MSCs. It is also feasible that loading would act to regulate the transport of chondrogenic growth factors within the regenerating tissue. For such reasons, in vitro models, as described in the proceeding sections, play a key role in improving our understanding of MSC mechanobiology.

\section{Role of cell shape and substrate stiffness in regulating stem cell differentiation}

Cell shape is determined by the internal organization of cytoskeleton as well as by interactions between adjacent cells and the surrounding matrix. It has been demonstrated that cell shape regulates commitment of human MSCs to the adipocyte or osteoblast lineage (McBeath et al., 2004). MSCs that were allowed to flatten and spread underwent osteogenesis, while unspread, round cells underwent adipogenesis. This lineage commitment is mediated through the RhoA-ROCK signalling pathway, a key regulator of the actin cytoskeleton, with RhoA and Rho kinase (ROCK) activity greater in spread cells than unspread cells. Inactivating RhoA promoted adipogenesis while activating it induced osteogenesis. RhoA signalling was shown to be dependant on cell shape, as expressing constitutively active RhoA in MSCs only induced osteogenesis in spread cells, see Fig. 2. In contrast, constitutive activation of ROCK, the downstream Rho effector, induced osteogenesis in both round and spread cells.

Cell shape and RhoA-Rock signalling may also regulate chondrogenesis of MSCs. In developing limbs, MSCs aggregate, resulting in increased cell density and cell-cell contact. As these cells undergo chondrogenesis they acquire a distinct spherical morphology and initiate expression of chondrogenic transcription factors 
Sox 5, Sox6 and Sox9. In monolayer culture of embryonic mesenchymal limb bud cells and ATDC5 cells, inhibition of ROCK with a pharmacological compound was shown increase expression of SOX9 and its target genes collagen II and aggrecan (Woods et al., 2005; Woods and Beier 2006). In addition, treatment with cytochalasin, a cytoskeleton-interrupting reagent, promotes chondrogenesis of embryonic mesenchymal cells (Zanetti and Solursh 1984) and bone marrow stem cells(Lim et al., 2000). Similarly, treatment of murine embryonic stem cells with cytochalasin leads to actin filament disruption and subsequent reorganization into a peripheral distribution which results in a more rounded morphology (Zhang et al., 2006). The expression of type II collagen, Sox9, and at a later time point, aggrecan was also up-regulated (Zhang et al., 2006). However in micromass culture, which better mimics development, primary limb bud cells display reduced expression of Sox9 target genes following ROCK inhibition despite increased levels of Sox9 mRNA expression (Woods and Beier 2006). A possible explanation for the discrepancy between two and three dimensional culture systems are the differences in cell-cell, cell-matrix interactions and cell shape that may regulate RhoA-ROCK signalling. Promotion of actin polymerisation using jasplakinolide treatment (which stabilizes existing actin filaments and thus blocks actin dynamics), enhances expression of chondrocyte marker genes in both monolayer and micromass culture (Woods and Beier 2006). While RhoA may inhibit chondrogenesis, overexpression of another small GTPase, Rac1, leads to increased N-cadherin (involved in cell-cell contacts) expression and mRNA transcripts of Sox5, SOX6, and SOX9, collagen II, and aggrecan(Woods et al., 2007).

Changes in the shape of the cell nucleus have also been shown to be accompanied by changes in SOX9 and aggrecan gene expression (McBride and Knothe Tate 2008). MSC nuclei tend to acquire a more rounded morphology as they proliferate to a high density, and this rounding is accompanied by increases in Runx2, Sox9, and Aggrecan gene expression (McBride and Knothe Tate 2008).

MSC differentiation is also directed by the local matrix stiffness (Engler et al., 2006). On soft collagen coated gels that mimic brain elasticity (0.1-1 kPa), MSCs tend to adhere, spread, and exhibit a neurogenic morphology, with associated up-regulation of neurogenic transcripts, see Fig 3. MSCs cultured on 10-fold stiffer matrices (8$17 \mathrm{kPa}$ ) become spindle-shaped in morphology, similar to myoblasts, with higher expression of myogenic message. On matrices $(25-40 \mathrm{kPa})$ that mimic the stiffness of 
osteoid, the MSC morphology becomes osteoblast-like, with greater expression of osteogenic genes. Nonmuscle myosin (NMM) II, which acts to tension the actin structures of the cytoskeleton, regulates this response. Addition of an inhibitor of NMM during MSC plating blocks the branching, elongation and spreading of the cell on any substrate, suggesting that NMM II activity is necessary for matrix-elasticitydriven differentiation of MSCs (Engler et al., 2006). As the matrix stiffness increases, myosin expression also increases, as well as the expression of focal adhesion components. A result of this is that cell stiffness increases as the matrix stiffness increases (Engler et al., 2006).

\section{Extrinsic Mechanical Signals as Regulators of MSC Fate}

The previous section highlighted the importance of RhoA-ROCK signalling in regulating differentiation of MSCs. Among other functions in the cell, ROCK mediates actin cytoskeletal tension and stress fibre formation, generating a state of pre-stress within the actin network of the cytoskeleton. These studies considered the role of intrinsic forces in regulating MSC differentiation, however extrinsic mechanical signals generated in the extracellular environment can also regulate MSC fate.

\section{Fluid Flow}

It has been demonstrated that MSCs respond to oscillatory fluid flow (OFF) with transient increases in intracellular $\mathrm{Ca}^{2+}$, increased proliferation rate, upregulation in osteoblastic gene expression, and decreased alkaline phosphatase activity( $\mathrm{Li}$ et al., 2004). OFF has also been shown to upregulate the expression of Runx2, Sox 9 and PPAR $\gamma$ (a marker of adipogenesis) in murine C3H10T1/2 MSCs, indicating that signals generated by fluid flow have the potential to regulate the expression of transcription factors involved in multiple differentiation pathways(Arnsdorf et al., 2009). In this study (Arnsdorf et al., 2009) it was demonstrated that mechanical stimulation via fluid flow activates RhoA and ROCKII, which may ultimately enhance tension in the actin cytoskeleton and which has previously been shown to regulate MSC differentiation (McBeath et al., 2004). Activating RhoA activity with lysophosphatidic acid sodium salt (LPA) treatment was shown to result in a twofold increase in Runx2 expression, which was further upregulated in response to fluid flow 
(Arnsdorf et al., 2009). Inhibiting ROCKII with Y27632 treatment, which also results in a loss of cytoskeletal integrity, abrogated flow-induced Runx2 upregulation, demonstrating that active ROCKII and an intact, dynamic actin cytoskeleton are necessary for flow-induced osteogenic differentiation (Arnsdorf et al., 2009), see Figure 4.

Fluid flow also regulates canonical and non-canonical Wnt signalling pathways in murine C3H10T1/2 MSCs (Arnsdorf et al., 2009). While the role of canonical Wnt signalling was outlined above, aspects of the non-canonical Wnt signalling pathway (which does not involve $\beta$-catenin) have the potential to initiate RhoA signalling among others (Katoh 2005; Katoh 2006). Nuclear $\beta$-catenin levels were significantly increased in MSCs in response to fluid flow, as were several Wnt associated proteins (Arnsdorf et al., 2009). The increase in fluid flow induced Runx2 expression in these cells was shown to be dependent on both Wnt5a (a Wnt with the potential to activate both canonical and non-canonical signalling (Mikels and Nusse 2006)) and $\beta$-catenin signalling. It was further demonstrated that flow-induced Wnt5 signalling was activating the non-canonical pathway, as Wnt5 signalling was not necessary for flow induced increases in nuclear $\beta$-catenin levels. Together these results demonstrate that both Wnt5a non-canonical signalling and $\beta$-catenin signalling are necessary for flow induced osteogenic differentiation. They also show that multiple pathways must be initiated for alterations in cell fate (Arnsdorf et al., 2009). Interestingly, it was also demonstrated that $\beta$-catenin signalling may be at least partially regulated by cadherin-catenin signalling. Cadherins are adhesion molecules involved in cell-cell contact. Fluid flow was shown to lead to a significant decrease in catenin-cadherin association, which could lead to increases in cytoplasmic $\beta$-catenin levels by liberating it from adherens junctions or cell-cell contacts, see Fig. 5. This suggests the intriguing possibility that adherens junctions may act as important mechanosensors in MSCs (Arnsdorf et al., 2009).

Fluid flow has been shown to up-regulate SOX9 expression in murine C3H/10T1/2 MSCs plated onto glass slides (Arnsdorf et al., 2009). Similar to previous findings(Woods et al., 2005), the authors of this study demonstrated that inhibiting tension within the actin cytoskeleton increased SOX9 expression. In addition, flow-induced SOX9 upregulation was abrogated by ROCKII inhibition, myosin II inhibition, actin polymerisation inhibition or actin stabilization, suggesting an intact and dynamic cytoskeleton is necessary for flow-induced chondrogenesis of 
MSCs (Arnsdorf et al., 2009). It has also been demonstrated that the response of murine MSCs depends on the cell seeding density (McBride et al., 2008). The up regulation of Sox 9 gene expression observed in these cells as they achieve a very high density through population doublings is silenced by the application of fluid flow, while SOX9 expression in cells plated directly at a very high density is increased in response to flow (McBride et al., 2008). This suggests that differences in cell shape regulate the response of these cells to flow.

\section{Tension}

Tensile strain has been shown to upregulate BMP-2 expression in MSCs embedded 3D collagen matrices, suggesting that strain alone can induce osteogenic differentiation (Sumanasinghe et al., 2006). It has also been demonstrated that bone marrow derived MSCs embedded in alginate gels respond differentially to the application of dynamic tension and compression (Haudenschild et al., 2009). Tension was observed to regulate both fibroblastic and osteogenic associated genes, while compression up-regulated chondrogenic gene expression (Haudenschild et al., 2009). It was suggested that tension inhibits chondrogenesis through increases in $\beta$-catenin signalling. Tensile strain can also regulate chondrogenic differentiation and glycosaminoglycan synthesis of MSCs embedded in collagen-GAG scaffolds(McMahon et al., 2008; McMahon et al., 2008). It has also been demonstrated that the application of tensile strains of $7.5 \%$ or greater induces apoptosis of MSCs seeded onto 2D silicone membranes (Kearney et al., 2008).

\section{Compression}

Huang et al. (2004) have demonstrated that mechanical signals induce chondrogenesis of bone-marrow derived MSCs as effectively as growth factor (TGF- $\beta 1$ ) stimulation. Rabbit MSCs were suspended in agarose hydrogels and subjected to cyclic compressive loading in the presence of absence of TGF- $\beta 1$. Aggrecan and collagen II gene expression were similar in the TGF- $\beta$ group and the loaded group, while combining loading with TGF- $\beta$ treatment promoted collagen II gene expression more effectively than TGF- $\beta 1$ alone. TGF- $\beta 1$ treatment and cyclic compression were both shown to upregulate TGF- $\beta 1$ gene expression, suggesting that both stimuli induce chondrogenesis of BM-MSCs through a similar pathway (Huang et al., 2004). In a 
follow-up study, cyclic compressive loading also promoted gene expression and protein production of two transmembrane TGF- $\beta$ receptors essential for chondrogenesis of MSCs when TGF- $\beta$ signalling is involved (Huang et al., 2005). This study also demonstrated that dynamic compression up-regulated both Sox-9 and TGF- $\beta 1$ at the gene and protein level. Finite element modelling was also used to determine the mechanical environment within such compressed agarose hydrogels (Huang et al., 2004), revealing that the hydrostatic pressure was low $(0.1 \mathrm{kPa})$, while compressive strain was similar in magnitude to that shown to enhance the biosynthetic activity of mature chondrocytes (Sah et al., 1989; Buschmann et al., 1995; Lee and Bader 1997; Mauck et al., 2000).

Dynamic compressive loading has also been shown to regulate chondrogenic gene expression in human bone marrow derived MSCs (Campbell et al., 2006; Pelaez et al., 2009). In the absence of chondrogenic growth factors, dynamic compression of MSCs embedded in alginate gels has been shown to down regulate collagen type II gene expression and upregulate aggrecan expression after 8 days (Campbell et al., 2006). Dynamic loading of bovine MSCs embedded in agarose hydrogels has also been shown to increase aggrecan promoter activity in both the core and annulus of the constructs (Mauck et al., 2007). As part of the same study it was demonstrated that sGAG accumulation was higher in dynamically compressed constructs in the absence of chondrogenic growth factors. However constructs maintained in free swelling conditions in the presence of TGF- $\beta 3$ accumulated significantly more sGAG. Similar studies using alternative loading regimes have also confirmed that while loading in the absence of TGF- $\beta$ enhances sGAG accumulation, levels are lower than that observed under free swelling conditions with TGF- $\beta$ supplementation (Kisiday et al., 2009). Static compressive loading has also been shown to enhance chondrogenesis of mouse embryonic limb bud mesenchymal cells embedded in collagen gels (Takahashi et al., 1998).

A more complex pattern emerges when MSCs are subjected to simultaneous compression and TGF- $\beta$ stimulation. The expression levels of aggrecan are reduced when MSCs are subjected to TGF- $\beta 3$ stimulation in addition to dynamic loading when compared to the action of the latter alone (Campbell et al., 2006). Longer term studies have also revealed that cartilage matrix production by MSCs embedded in agarose hydrogels is reduced when mechanical stimulation is initiated at the onset of TGF- $\beta$ induced chondrogenesis (Campbell et al., 2006; Thorpe et al., 2008). In 
contrast, cyclic compression enhances proteoglycan and collagen accumulation for MSCs embedded in hyaluronan-gelatin composites (Angele et al., 2004). Such contradictory findings suggest that the interaction of MSCs with their surrounding matrix is critical to their response to loading. Related to this is the observation that the response of MSCs to dynamic compression in the presence of TGF- $\beta 1$ depends on when loading is initiated; application at early time-points (day 8) decreases aggrecan gene expression; while loading at later time-points (day 16) increases chondrogenic gene expression (Mouw et al., 2007). This suggests that the mechano-responsiveness of MSCs changes depending on the stage of chondrogenesis and the development of a pericellular matrix. Compressive loading may also play a role in the maintenance of the chondrogenic phenotype following the withdrawal of TGF- $\beta$ supplementation. For example, sGAG accumulation is enhanced in MSC laden constructs by the application of dynamic compression following 3 weeks of free swelling culture in the presence of TGF- $\beta$ (in review). Similarly following 7 days of pre-culture in free swelling conditions fibrin-polyurethane composite scaffolds, various mechanical loading protocols incorporating dynamic compression induced higher glycosaminoglycan synthesis and higher chondrocytic gene expression ( $\mathrm{Li}$ et al., 2009). The same authors have also demonstrated that the influence of mechanical loading depends on the TGF- $\beta$ concentration of the culture medium, with a much stronger effect on gene expression observed at lower TGF- $\beta 1$ concentrations (Li et al., 2009). When TGF- $\beta 1$ was absent from the medium, mechanical load stimulated mRNA expression and protein synthesis of TGF- $\beta 1$ and TGF- $\beta 3$ (Li et al., 2009).

\section{Hydrostatic Pressure}

The application of cyclic hydrostatic pressure has been shown to enhance chondrogenesis of MSC aggregates maintained in a defined chondrogenic culture medium, as evidenced by increases in proteoglycan and collagen content (Angele et al., 2003). Application of cyclic hydrostatic pressure has also been shown to enhance Sox 9 mRNA expression as well as type II collagen and aggrecan mRNA expression in MSC aggregates maintained in chondrogenic conditions compared to unloaded controls (Miyanishi et al., 2006). Interestingly, this study also demonstrated that the application of cyclic hydrostatic pressure in the absence of TGF- $\beta 3$ also up regulated Sox9, type II collagen and aggrecan expression, however expression levels were 
lower than for unloaded aggregates supplemented with TGF- $\beta 3$ (Miyanishi et al., 2006). In a separate study, the same authors demonstrated that the magnitude of hydrostatic pressure $(0.1,1$ or $10 \mathrm{MPa})$ differentially regulated chondrogenesis of MSC aggregates, with greater type II collagen mRNA expression and collagen accumulation at $10 \mathrm{MPa}$ (Miyanishi et al., 2006). Matrix condensation also increases with increasing levels of hydrostatic pressure (Miyanishi et al., 2006). Sox9 expression has been shown to transiently increase in bone marrow derived MSCs embedded in agarose hydrogels, however this did not lead to increases in aggrecan and collagen II mRNA expression(Finger et al., 2007). This is in contrast to previously described increases observed in MSC aggregates (Miyanishi et al., 2006), which may be due to greater cell-cell proximity in these pellets compared to agarose hydrogels (Finger et al., 2007). In bone marrow derived MSCs embedded in type I collagen sponges and subjected to hydrostatic pressure in a media containing osteogenic and chondrogenic factors, mRNA expression of chondrogenic genes such as aggrecan, type II collagen and Sox9 increased, with no change in the expression of the osteogenic marker Runx2 (Wagner et al., 2008). Significantly lower magnitudes of dynamic pressure have also been shown to promote chondrogenesis of MSCs embedded in alginate beads, and this response was at least partially regulated via activated phosphorylation of p38 MAPK (Li et al., 2009). In contrast, it has been reported that hydrostatic pressure has little or no effect on chondrogenic gene expression or matrix accumulation in MSC aggregates, in either the presence or absence of TGF- $\beta$ or BMP-2(Zeiter et al., 2009).

\section{Conclusions}

The role of mechanical signals in regulating MSC behaviour and fate is much less studied and understood than biochemical stimuli. This is perhaps surprising considering the critical role mechanics is known to have during development, bone and joint formation and in regenerative events such as fracture healing. It is highly likely that mechanical signals work in a synergistic way with other cues to regulate and coordinate MSC behaviour and function and this is even less understood than the effect of mechanical signalling alone. Furthermore, the majority of MSC mechanobiology studies to date have considered the roles of intrinsic and extrinsic forces in regulating MSC fate separately. Some of the few studies that have considered both cytoskeletal organization and externally applied mechanical signals 
suggest that factors such as cell shape, cytoskeletal dynamics, cell-cell and cell-matrix interactions will determine how MSCs respond to extrinsic signals (both biomechanical and biochemical) from their local environment. As the mechanical regulation of MSC differentiation is starting to be better understood, further research effort is also required to elucidate the molecular mechanisms responsible for cellular mechanotransduction. Investigating the role of potential mechano-sensors such as stretch-activated channels, primary cilia and various transmembrane and cytoskeleton-associated proteins in MSCs represents the first step in this grand challenge, which will ultimately improve our understanding of the role of MSCs in disease and their potential for regenerative therapy in medicine.

\section{Acknowledgements}

This manuscript was written while DK was a visiting research scholar at Columbia University. Funding for this was provided by the Fulbright Program and Science Foundation Ireland under the President of Ireland Young Researcher Award. 


\section{Figures}

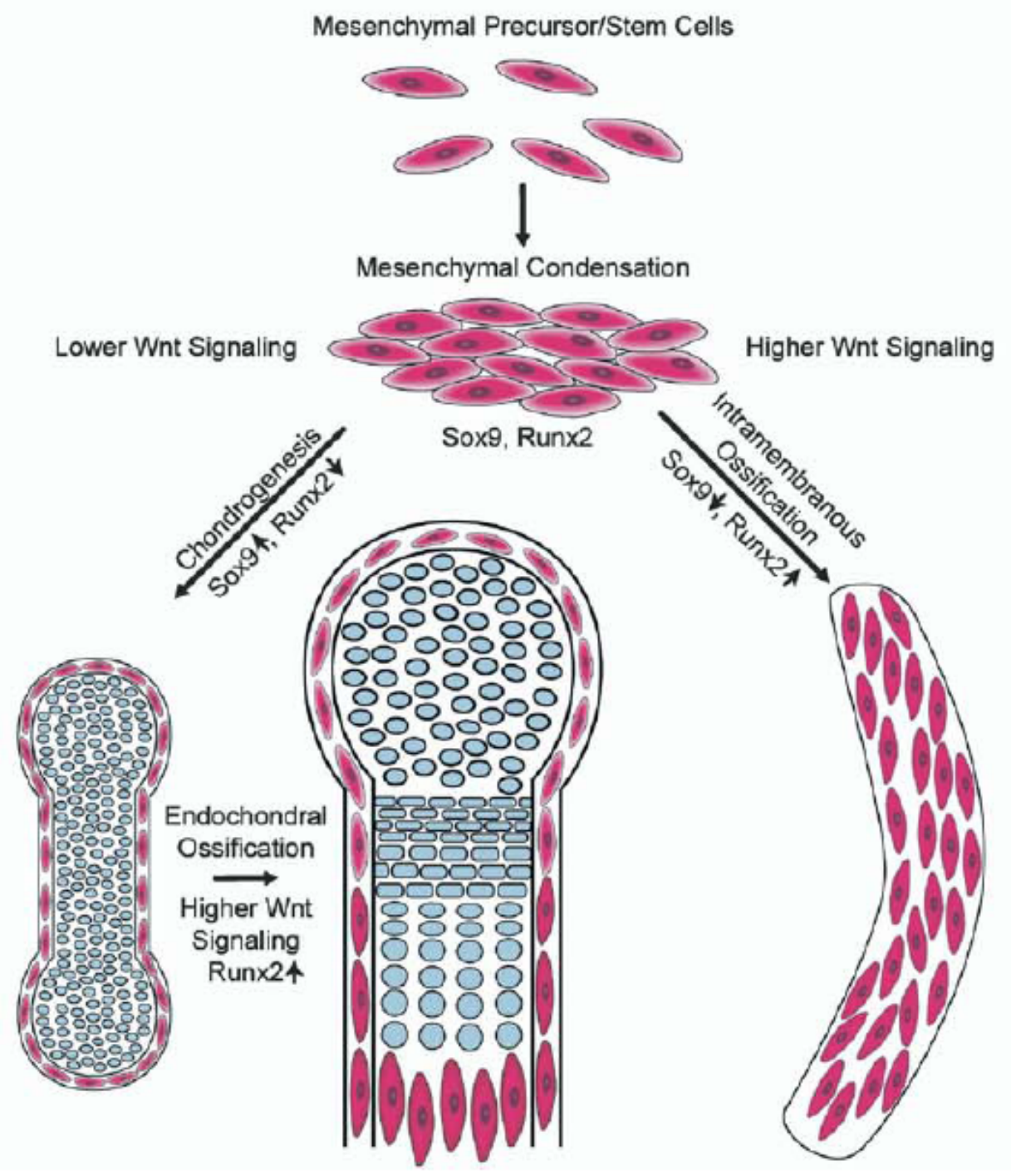

Figure 1. Proposed model for the role of Wnt signalling in controlling chondrogenesis and osteogenesis of stem cells in mesenchymal condensations(Day et al., 2005). Canonical Wnt signalling promotes Runx2 expression and prevents chondrocyte differentiation during intramembranous ossification by inhibiting Sox 9 expression. Canonical Wnt signalling is inhibited during endochondral ossification, leading to upregulation of Sox9 expression and downregulation of Runx2 expression. In the later stages of endochondral ossification, Runx 2 expression is upregulated in 
the perichondrium by canonical Wnt signalling leading to the formation of the bone collar. Adapted from (Day et al., 2005). PREMISSION NOT RECEIVED.

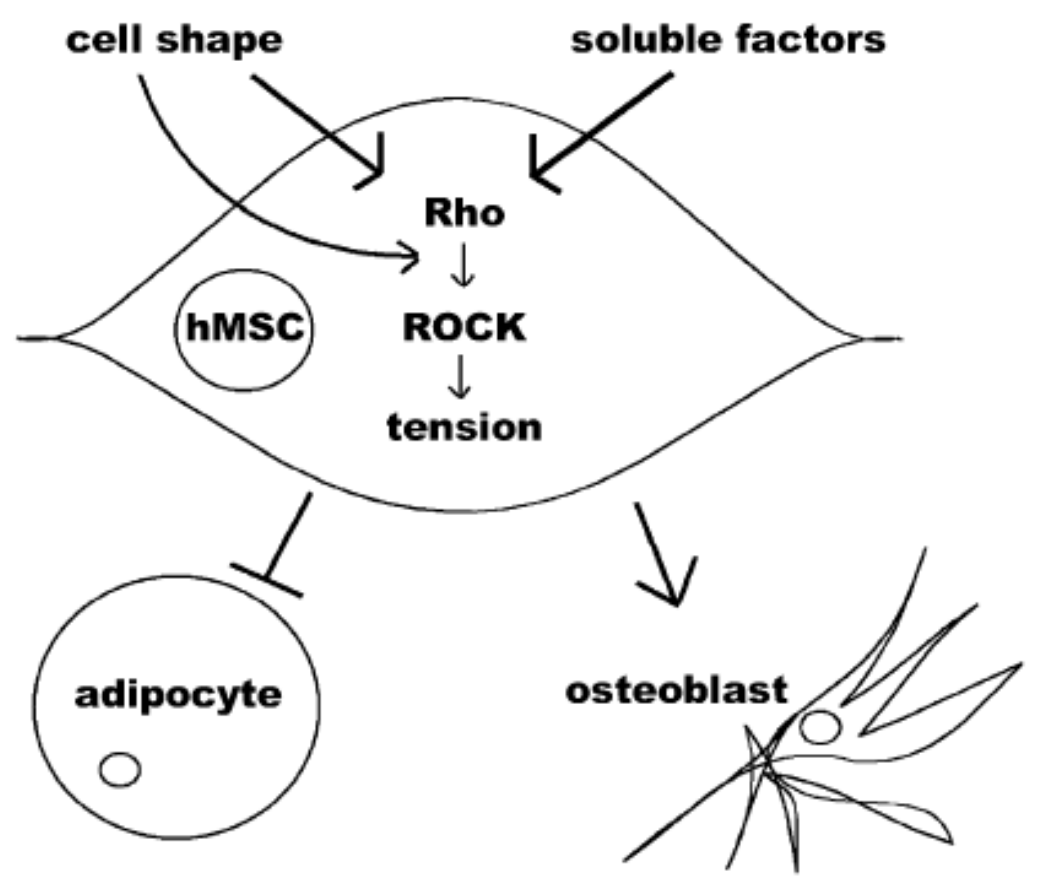

Figure 2. Cell shape acts as a mechanical cue, driving hMSC commitment between the adipocyte and osteoblast phenotype when RhoA signaling and cytoskeletal tension are intact. Interference with cell shape, RhoA signaling, ROCK activity, or cytoskeletal tension alters hMSC commitment. Adapted from (McBeath et al., 2004). PREMISSION NOT RECEIVED. 

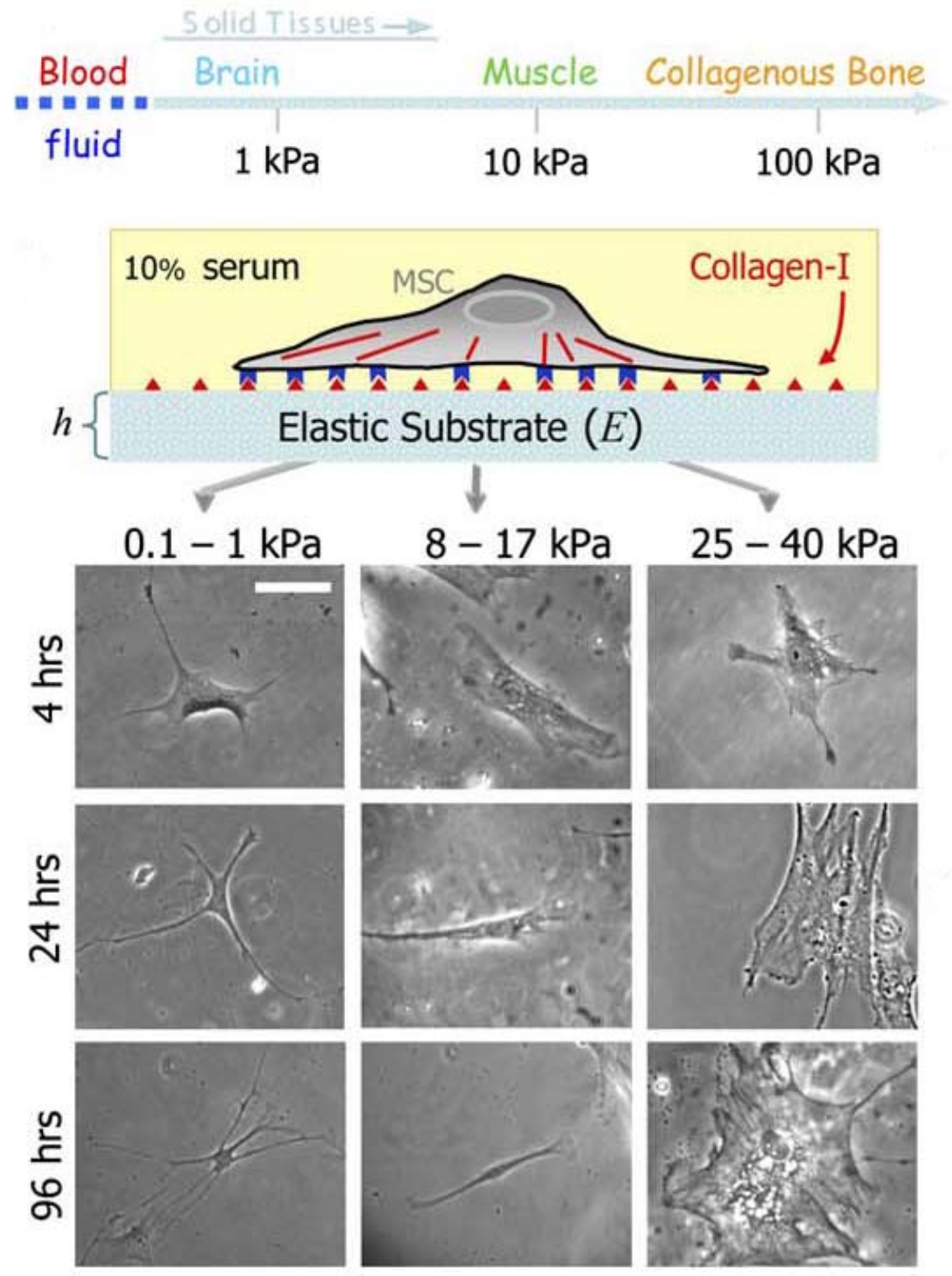

Figure 3. Matrix elasticity regulates stem cell differentiation(Engler et al., 2006). MSCs are platted onto substrates of varying stiffness. On soft, collagen-coated gels that mimic brain elasticity $(0.1-1 \mathrm{kPa})$, the vast majority of MSCs adhere, spread, and exhibit an increasingly branched, filopodia-rich morphology. MSCs on 10-fold stiffer matrices that mimic striated muscle elasticity $(8-17 \mathrm{kPa})$ lead to spindle-shaped cells similar in shape to myoblasts. Stiffer matrices $(25-40 \mathrm{kPa})$ that mimic the crosslinked collagen of osteoids yield polygonal MSCs similar in morphology to osteoblasts. Adapted from (Engler et al., 2006). PREMISSION NOT RECEIVED. 
A
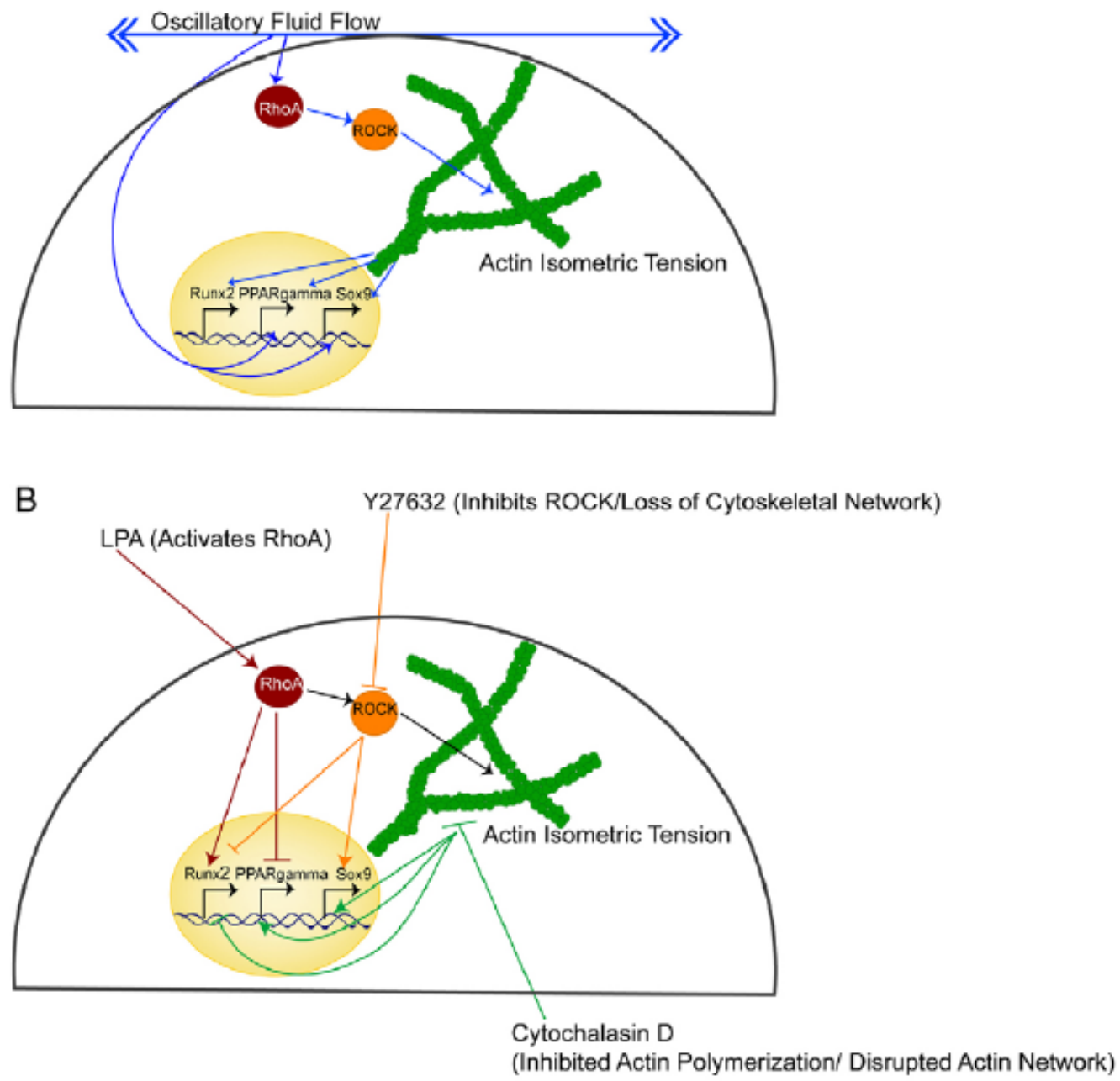

Figure 4. A. Exposure to fluid flow regulates MSC differentiation by activating the RhoA/RockII pathway. Tension within the actin cytoskeleton is required for the activation of mechanosensors or signalling mechanisms involved in the transduction of dynamic fluid flow into cell fate decisions. Fluid flow may have the potential to regulate multiple transcription factors involved in unique lineage pathways. B. Rock II activity and a dynamic actin cytoskeleton under tension is required for Runx2 expression. RhoA activation induces Runx2 expression, downregulates PPAR $\gamma$ and does not alter Sox9 expression. While biochemical disruption of the actin network differentially regulated the expression of these transcription factors, in all cases an intact actin network was necessary for flow-induced gene expression. Adapted from (Arnsdorf et al., 2009). PREMISSION NOT RECEIVED. 


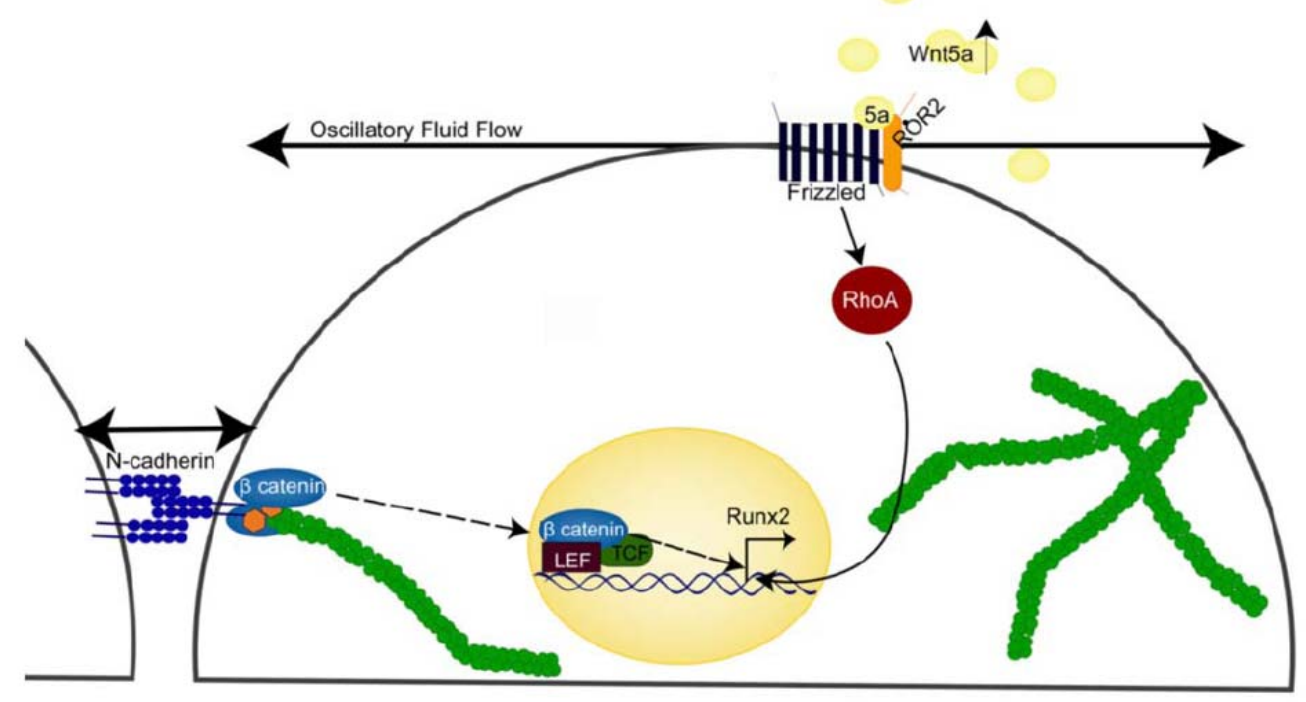

Figure 5. Fluid flow has the potential to regulate the non-canonical Wnt5a and $\beta$ catenin signalling pathways in MSCs, both of which are essential for flow induced osteogenic differentiation. $\beta$-catenin signalling appears to be mediated at least in part by alterations in $\mathrm{N}$-cadherin/ $\beta$-catenin association in response to fluid flow, suggesting that adherens junctions are involved in mechano-transduction in MSCs. Adapted from(Arnsdorf et al., 2009). PREMISSION NOT RECEIVED. 


\section{References}

Angele, P., Schumann, D., Angele, M., Kinner, B., Englert, G., Hente, R., Fu?chtmeier, B., Nerlich, M., Neumann, C. and Kujat, R. (2004). Cyclic, mechanical compression enhances chondrogenesis of mesenchymal progenitor cells in tissue engineering scaffolds. Biorheology 41(3-4): 335-346.

Angele, P., Yoo, J. U., Smith, C., Mansour, J., Jepsen, K. J., Nerlich, M. and Johnstone, B. (2003). Cyclic hydrostatic pressure enhances the chondrogenic phenotype of human mesenchymal progenitor cells differentiated in vitro. Journal of Orthopaedic Research 21(3): 451-457.

Arnsdorf, E. J., Tummala, P. and Jacobs, C. R. (2009). Non-canonical Wnt signalling and $\mathrm{N}$-cadherin related $\hat{\mathrm{I}}^{2}$-catenin signalling play a role in mechanically induced osteogenic cell fate. PLOS ONE 4(4).

Arnsdorf, E. J., Tummala, P., Kwon, R. Y. and Jacobs, C. R. (2009). Mechanically induced osteogenic differentiation - The role of RhoA, ROCKII and cytoskeletal dynamics. Journal of Cell Science 122(4): 546-553.

Bi, W., Deng, J. M., Zhang, Z., Behringer, R. R. and De Crombrugghe, B. (1999). Sox9 is required for cartilage formation. Nature Genetics 22(1): 85-89.

Boccaccio, A., Pappalettere, C. and Kelly, D. J. (2007). The influence of expansion rates on mandibular distraction osteogenesis: A computational analysis. Annals of Biomedical Engineering 35(11): 1940-1960.

Boccaccio, A., Prendergast, P. J., Pappalettere, C. and Kelly, D. J. (2008). Tissue differentiation and bone regeneration in an osteotomized mandible: A computational analysis of the latency period. Medical and Biological Engineering and Computing 46(3): 283-298.

Buschmann, M. D., Gluzband, Y. A., Grodzinsky, A. J. and Hunziker, E. B. (1995). Mechanical compression modulates matrix biosynthesis in chondrocyte/agarose culture. J Cell Sci 108 ( Pt 4): 1497-508.

Campbell, J. J., Lee, D. A. and Bader, D. L. (2006). Dynamic compressive strain influences chondrogenic gene expression in human mesenchymal stem cells. Biorheology 43(3-4): 455-470.

Carter, D. R., BeauprÃ®), G. S., Giori, N. J. and Helms, J. A. (1998). Mechanobiology of skeletal regeneration. Clinical Orthopaedics and Related Research(355 SUPPL.): S41-S55.

Chao, E. Y. S., Inoue, N., Elias, J. J. and Aro, H. (1998). Enhancement of fracture healing by mechanical and surgical intervention. Clinical Orthopaedics and Related Research(355 SUPPL.): S163-S178.

Day, T. F., Guo, X., Garrett-Beal, L. and Yang, Y. (2005). Wnt $/ \hat{\mathrm{I}}^{2}$-catenin signaling in mesenchymal progenitors controls osteoblast and chondrocyte differentiation during vertebrate skeletogenesis. Developmental Cell 8(5): 739-750.

Day, T. F. and Yang, Y. (2008). Wnt and hedgehog signaling pathways in bone development. Journal of Bone and Joint Surgery - Series A 90(SUPPL. 1): 1924.

Ducy, P., Zhang, R., Geoffroy, V., Ridall, A. L. and Karsenty, G. (1997). Osf2/Cbfa1: A transcriptional activator of osteoblast differentiation. Cell 89(5): 747-754.

Elder, S. H., Goldstein, S. A., Kimura, J. H., Soslowsky, L. J. and Spengler, D. M. (2001). Chondrocyte differentiation is modulated by frequency and duration of cyclic compressive loading. Ann Biomed Eng 29(6): 476-82.

Elder, S. H., Kimura, J. H., Soslowsky, L. J., Lavagnino, M. and Goldstein, S. A. (2000). Effect of compressive loading on chondrocyte differentiation in agarose cultures of chick limb-bud cells. J Orthop Res 18(1): 78-86. 
Engler, A. J., Sen, S., Sweeney, H. L. and Discher, D. E. (2006). Matrix elasticity directs stem cell lineage specification. Cell 126(4): 677-89.

Estes, B. T., Gimble, J. M. and Guilak, F. (2004). Mechanical Signals as Regulators of Stem Cell Fate. Current Topics in Developmental Biology. 60: 91-126.

Ferguson, C., Alpern, E., Miclau, T. and Helms, J. A. (1999). Does adult fracture repair recapitulate embryonic skeletal formation? Mechanisms of Development 87(1-2): 57-66.

Finger, A. R., Sargent, C. Y., Dulaney, K. O., Bernacki, S. H. and Loboa, E. G. (2007). Differential effects on messenger ribonucleic acid expression by bone marrow-derived human mesenchymal stem cells seeded in agarose constructs due to ramped and steady applications of cyclic hydrostatic pressure. Tissue Engineering 13(6): 1151-1158.

Glucksmann, A. (1942). The role of mechanical stresses in bone formation in vitro. $J$ Anat 76: 231-239.

Glucksmann, A. (1939). Studies on bone mechanics in vitro: II. The role of tension and pressure in chondrogenesis. Anat Rec 73: 39-56.

Goldring, M. B., Tsuchimochi, K. and Ijiri, K. (2006). The control of chondrogenesis. Journal of Cellular Biochemistry 97(1): 33-44.

Goodship, A. E., Cunningham, J. L. and Kenwright, J. (1998). Strain rate and timing of stimulation in mechanical modulation of fracture healing. Clinical Orthopaedics and Related Research(355 SUPPL.): S105-S115.

Goodship, A. E. and Kenwright, J. (1985). The influence of induced micromovement upon the healing of experimental tibial fractures. Journal of Bone and Joint Surgery - Series B 67(4): 650-655.

Goodship, A. E., Lawes, T. J. and Rubin, C. T. (2009). Low-magnitude highfrequency mechanical signals accelerate and augment endochondral bone repair: Preliminary evidence of efficacy. Journal of Orthopaedic Research 27(7): 922-930.

Gordon, M. D. and Nusse, R. (2006). Wnt signaling: Multiple pathways, multiple receptors, and multiple transcription factors. Journal of Biological Chemistry 281(32): 22429-22433.

Hall, B. K. (1968). In vitro studies on the mechanical evocation of advenitious cartilage in the chick. Journal of Experimental Zoology 168(3): 283-305.

Hall, B. K. (1972). Immobilization and cartilage transformation into bone in the embryonic chick. Anatomical Record 173(4): 391-403.

Hall, B. K. (1979). Selective proliferation and accumulation of chondroprogenitor cells as the mode of action of biomechanical factors during secondary chondrogenesis. Teratology 20(1): 81-92.

Hall, B. K. and Herring, S. W. (1990). Paralysis and growth of the musculoskeletal system in the embryonic chick. Journal of Morphology 206(1): 45-56.

Haudenschild, A. K., Hsieh, A. H., Kapila, S. and Lotz, J. C. (2009). Pressure and distortion regulate human mesenchymal stem cell gene expression. Annals of Biomedical Engineering 37(3): 492-502.

Henderson, J. H. and Carter, D. R. (2002). Mechanical induction in limb morphogenesis: The role of growth-generated strains and pressures. Bone 31(6): 645-653.

Hosseini, A. and Hogg, D. A. (1991). The effects of paralysis on skeletal development in the chick embryo: I. General effects. Journal of Anatomy 177: 159-168. 
Hosseini, A. and Hogg, D. A. (1991). The effects of paralysis on skeletal development in the chick embryo: II. Effects on histogenesis of the tibia. Journal of Anatomy 177: 169-178.

Huang, C. Y. C., Hagar, K. L., Frost, L. E., Sun, Y. and Cheung, H. S. (2004). Effects of cyclic compressive loading on chondrogenesis of rabbit bone-marrow derived mesenchymal stem cells. Stem Cells 22(3): 313-323.

Huang, C. Y. C., Reuben, P. M. and Cheung, H. S. (2005). Temporal expression patterns and corresponding protein inductions of early responsive genes in rabbit bone marrow-derived mesenchymal stem cells under cyclic compressive loading. Stem Cells 23(8): 1113-1121.

Isaksson, H., van Donkellar, C. C., Huiskes, R. and Ito, K. (2006). Corroboration of mechanoregulatory algorithms for tissue differentiation during fracture healing: Comparison with in vivo results. Journal of Orthopaedic Research 24(5): 898-907.

Isaksson, H., Wilson, W., van Donkelaar, C. C., Huiskes, R. and Ito, K. (2006). Comparison of biophysical stimuli for mechano-regulation of tissue differentiation during fracture healing. Journal of Biomechanics 39(8): 15071516.

Karsenty, G. and Wagner, E. F. (2002). Reaching a genetic and molecular understanding of skeletal development. Developmental Cell 2(4): 389-406.

Katoh, M. (2005). WNT/PCP signaling pathway and human cancer (review). Oncology reports. 14(6): 1583-1588.

Katoh, M. (2006). Cross-talk of WNT and FGF signaling pathways at GSK3 $\hat{\mathrm{I}}^{2}$ to regulate $\hat{\mathrm{I}}^{2}$-catenin and SNAIL signaling cascades. Cancer Biology and Therapy 5(9): 1059-1064.

Kearney, E. M., Prendergast, P. J. and Campbell, V. A. (2008). Mechanisms of strainmediated mesenchymal stem cell apoptosis. Journal of Biomechanical Engineering 130(6).

Kelly, D. J. and Prendergast, P. J. (2005). Mechano-regulation of stem cell differentiation and tissue regeneration in osteochondral defects. $J$ Biomech 38(7): 1413-22.

Kelly, D. J. and Prendergast, P. J. (2006). Prediction of the optimal mechanical properties for a scaffold used in osteochondral defect repair. Tissue Eng 12(9): 2509-19.

Kenwright, J. and Goodship, A. E. (1989). Controlled mechanical stimulation in the treatment of tibial fractures. Clinical Orthopaedics and Related Research (241): 36-47.

Kenwright, J., Richardson, J. B., Cunningham, J. L., White, S. H., Goodship, A. E., Adams, M. A., Magnussen, P. A. and Newman, J. H. (1991). Axial movement and tibial fractures: A controlled randomised trial of treatment. Journal of Bone and Joint Surgery - Series B 73(4): 654-659.

Kisiday, J. D., Frisbie, D. D., McIlwraith, C. W. and Grodzinsky, A. J. (2009). Dynamic compression stimulates proteoglycan synthesis by mesenchymal stem cells in the absence of chondrogenic cytokines. Tissue Engineering - Part A 15(10): 2817-2824.

Klein-Nulend, J., Veldhuijzen, J. P. and Burger, E. H. (1986). Increased calcification of growth plate cartilage as a result of compressive force in vitro. Arthritis and Rheumatism 29(8): 1002-1009. 
Knothe Tate, M. L., Falls, T. D., McBride, S. H., Atit, R. and Knothe, U. R. (2008). Mechanical modulation of osteochondroprogenitor cell fate. International Journal of Biochemistry and Cell Biology 40(12): 2720-2738.

Komori, T., Yagi, H., Nomura, S., Yamaguchi, A., Sasaki, K., Deguchi, K., Shimizu, Y., Bronson, R. T., Gao, Y. H., Inada, M., Sato, M., Okamoto, R., Kitamura, Y., Yoshiki, S. and Kishimoto, T. (1997). Targeted disruption of Cbfa1 results in a complete lack of bone formation owing to maturational arrest of osteoblasts. Cell 89(5): 755-764.

Lacroix, D. and Prendergast, P. J. (2002). A mechano-regulation model for tissue differentiation during fracture healing: Analysis of gap size and loading. Journal of Biomechanics 35(9): 1163-1171.

Lacroix, D. and Prendergast, P. J. (2002). Three-dimensional simulation of fracture repair in the human tibia. Computer methods in biomechanics and biomedical engineering 5(5): 369-376.

Lee, D. A. and Bader, D. L. (1997). Compressive strains at physiological frequencies influence the metabolism of chondrocytes seeded in agarose. Journal of Orthopaedic Research 15(2): 181-188.

Li, J., Zhao, Z., Yang, J., Liu, J., Wang, J., Li, X. and Liu, Y. (2009). p38 MAPK mediated in compressive stress-induced chondrogenesis of rat bone marrow MSCs in 3D alginate scaffolds. Journal of Cellular Physiology 221(3): 609617.

Li, Y. J., Batra, N. N., You, L., Meier, S. C., Coe, I. A., Yellowley, C. E. and Jacobs, C. R. (2004). Oscillatory fluid flow affects human marrow stromal cell proliferation and differentiation. Journal of Orthopaedic Research 22(6): 1283-1289.

Li, Z., Kupcsik, L., Yao, S. J., Alini, M. and Stoddart, M. J. (2009). Mechanical Load Modulates Chondrogenesis of Human Mesenchymal Stem Cells through the TGF-beta Pathway. J Cell Mol Med. 2009 May 11.

Li, Z., Yao, S. J., Alini, M. and Stoddart, M. J. (2009). Chondrogenesis of Human Bone Marrow Mesenchymal Stem Cells in Fibrin-Polyurethane Composites is Modulated by Frequency and Amplitude of Dynamic Compression and Shear Stress. Tissue Eng Part A.

Lim, Y. B., Kang, S. S., Park, T. K., Lee, Y. S., Chun, J. S. and Sonn, J. K. (2000). Disruption of actin cytoskeleton induces chondrogenesis of mesenchymal cells by activating protein kinase C-ÎI signaling. Biochemical and Biophysical Research Communications 273(2): 609-613.

Loboa, E. G., BeaupreÌ , G. S. and Carter, D. R. (2001). Mechanobiology of initial pseudarthrosis formation with oblique fractures. Journal of Orthopaedic Research 19(6): 1067-1072.

Mauck, R. L., Byers, B. A., Yuan, X. and Tuan, R. S. (2007). Regulation of cartilaginous ECM gene transcription by chondrocytes and MSCs in 3D culture in response to dynamic loading. Biomech Model Mechanobiol 6(1-2): 113-25.

Mauck, R. L., Soltz, M. A., Wang, C. C., Wong, D. D., Chao, P. H., Valhmu, W. B., Hung, C. T. and Ateshian, G. A. (2000). Functional tissue engineering of articular cartilage through dynamic loading of chondrocyte-seeded agarose gels. J Biomech Eng 122(3): 252-60.

McBeath, R., Pirone, D. M., Nelson, C. M., Bhadriraju, K. and Chen, C. S. (2004). Cell shape, cytoskeletal tension, and RhoA regulate stem cell lineage commitment. Developmental Cell 6(4): 483-495. 
McBride, S. H., Falls, T. and Knothe Tate, M. L. (2008). Modulation of stem cell shape and fate B: Mechanical modulation of cell shape and gene expression. Tissue Engineering - Part A. 14(9): 1573-1580.

McBride, S. H. and Knothe Tate, M. L. (2008). Modulation of stem cell shape and fate A: The role of density and seeding protocol on nucleus shape and gene expression. Tissue Engineering - Part A. 14(9): 1561-1572.

McMahon, L. A., Campbell, V. A. and Prendergast, P. J. (2008). Involvement of stretch-activated ion channels in strain-regulated glycosaminoglycan synthesis in mesenchymal stem cell-seeded 3D scaffolds. Journal of Biomechanics 41(9): 2055-2059.

McMahon, L. A., Reid, A. J., Campbell, V. A. and Prendergast, P. J. (2008). Regulatory effects of mechanical strain on the chondrogenic differentiation of MSCs in a collagen-GAG scaffold: Experimental and computational analysis. Annals of Biomedical Engineering 36(2): 185-194.

Mikels, A. J. and Nusse, R. (2006). Purified Wnt5a protein activates or inhibits betacatenin-TCF signaling depending on receptor context. PLoS biology 4(4).

Mikic, B., Isenstein, A. L. and Chhabra, A. (2004). Mechanical modulation of cartilage structure and function during embryogenesis in the chick. Annals of Biomedical Engineering 32(1): 18-25.

Mikic, B., Johnson, T. L., Chhabra, A. B., Schalet, B. J., Wong, M. and Hunziker, E. B. (2000). Differential effects of embryonic immobilization on the development of fibrocartilaginous skeletal elements. Journal of Rehabilitation Research and Development 37(2): 127-133.

Miyanishi, K., Trindade, M. C., Lindsey, D. P., Beaupre, G. S., Carter, D. R., Goodman, S. B., Schurman, D. J. and Smith, R. L. (2006). Dose- and timedependent effects of cyclic hydrostatic pressure on transforming growth factor-beta3-induced chondrogenesis by adult human mesenchymal stem cells in vitro. Tissue Eng 12(8): 2253-62.

Miyanishi, K., Trindade, M. C., Lindsey, D. P., Beaupre, G. S., Carter, D. R., Goodman, S. B., Schurman, D. J. and Smith, R. L. (2006). Effects of hydrostatic pressure and transforming growth factor-beta 3 on adult human mesenchymal stem cell chondrogenesis in vitro. Tissue Eng 12(6): 1419-28.

Mouw, J. K., Connelly, J. T., Wilson, C. G., Michael, K. E. and Levenston, M. E. (2007). Dynamic compression regulates the expression and synthesis of chondrocyte-specific matrix molecules in bone marrow stromal cells. Stem Cells 25(3): 655-663.

Murray, P. D. and Drachman, D. B. (1969). The role of movement in the development of joints and related structures: the head and neck in the chick embryo. Journal of Embryology and Experimental Morphology 22(3): 349-371.

Nagel, T. and Kelly, D. J. (2009). Mechano-regulation of mesenchymal stem cell differentiation and collagen organisation during skeletal tissue repair. Biomechanics and Modeling in Mechanobiology: 1-14.

Nowlan, N. C., Bourdon, C., Dumas, G., Tajbakhsh, S., Prendergast, P. J. and Murphy, P. Developing bones are differentially affected by compromised skeletal muscle formation. Bone.

Nowlan, N. C., Murphy, P. and Prendergast, P. J. (2007). Mechanobiology of embryonic limb development. Annals of the New York Academy of Sciences. 1101: 389-411.

Osborne, A. C., Lamb, K. J., Lewthwaite, J. C., Dowthwaite, G. P. and Pitsillides, A. A. (2002). Short-term rigid and flaccid paralyses diminish growth of 
embryonic chick limbs and abrogate joint cavity formation but differentially preserve pre-cavitated joints. Journal of Musculoskeletal Neuronal Interactions 2(5): 448-456.

Otto, F., Thornell, A. P., Crompton, T., Denzel, A., Gilmour, K. C., Rosewell, I. R., Stamp, G. W. H., Beddington, R. S. P., Mundlos, S., Olsen, B. R., Selby, P. B. and Owen, M. J. (1997). Cbfa1, a candidate gene for cleidocranial dysplasia syndrome, is essential for osteoblast differentiation and bone development. Cell 89(5): 765-771.

Pauwels, F. (1941). Grundrĩ̃ Ÿ einer Biomechanik der Frakturheilung. 34. Kongress

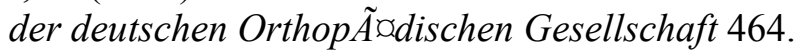

Pauwels, F. (1960). Eine neue Theorie ù̀`ber den EinfluÃ Ÿ mechanischer Reize auf die Differenzierung der StuÌ tzgewebe - Zehnter Beitrag zur funktionellen Anatomie und kausalen Morphologie des Stù̀^tzapparates. Zeitschrift fuÌ $r$ Anatomie und Entwicklungsgeschichte 121(6): 478-515.

Pelaez, D., Charles Huang, C. Y. and Cheung, H. S. (2009). Cyclic compression maintains viability and induces chondrogenesis of human mesenchymal stem cells in fibrin gel scaffolds. Stem Cells and Development 18(1): 93-102.

Perez-Moreno, M. and Fuchs, E. (2006). Catenins: Keeping Cells from Getting Their Signals Crossed. Developmental Cell 11(5): 601-612.

Petrovic, A. (1974). Control of postnatal growth of secondary cartilages of the mandible by mechanisms regulating occlusion. Cybernetic model. Transactions. European Orthodontic Society: 69-75.

Prendergast, P. J., Huiskes, R. and Søballe, K. (1997). Biophysical stimuli on cells during tissue differentiation at implant interfaces. Journal of Biomechanics 30(6): 539-548.

Sah, R. L. Y., Kim, Y. J., Doong, J. Y. H., Grodzinsky, A. J., Plaas, A. H. K. and Sandy, J. D. (1989). Biosynthetic response of cartilage explants to dynamic compression. Journal of Orthopaedic Research 7(5): 619-636.

Sumanasinghe, R. D., Bernacki, S. H. and Loboa, E. G. (2006). Osteogenic differentiation of human mesenchymal stem cells in collagen matrices: Effect of uniaxial cyclic tensile strain on bone morphogenetic protein (BMP-2) mRNA expression. Tissue Engineering 12(12): 3459-3465.

Takahashi, I., Nuckolls, G. H., Takahashi, K., Tanaka, O., Semba, I., Dashner, R., Shum, L. and Slavkin, H. C. (1998). Compressive force promotes sox9, type II collagen and aggrecan and inhibits IL-1beta expression resulting in chondrogenesis in mouse embryonic limb bud mesenchymal cells. $J$ Cell Sci 111 ( Pt 14): 2067-76.

Thorpe, S. D., Buckley, C. T., Vinardell, T., O'Brien, F. J., Campbell, V. A. and Kelly, D. J. (2008). Dynamic compression can inhibit chondrogenesis of mesenchymal stem cells. Biochemical and Biophysical Research Communications.

Vortkamp, A., Pathi, S., Peretti, G. M., Caruso, E. M., Zaleske, D. J. and Tabin, C. J. (1998). Recapitulation of signals regulating embryonic bone formation during postnatal growth and in fracture repair. Mechanisms of Development 71(1-2): 65-76.

Wagner, D. R., Lindsey, D. P., Li, K. W., Tummala, P., Chandran, S. E., Smith, R. L., Longaker, M. T., Carter, D. R. and Beaupre, G. S. (2008). Hydrostatic pressure enhances chondrogenic differentiation of human bone marrow stromal cells in osteochondrogenic medium. Annals of Biomedical Engineering 36(5): 813-820. 
Wong, M., Germiller, J., Bonadio, J. and Goldstein, S. A. (1993). Neuromuscular atrophy alters collagen gene expression, pattern formation, and mechanical integrity of the chick embryo long bone. Progress in clinical and biological research 383 B: 587-597.

Woods, A. and Beier, F. (2006). RhoA/ROCK signaling regulates chondrogenesis in a context-dependent manner. Journal of Biological Chemistry 281(19): 1313413140.

Woods, A., Wang, G. and Beier, F. (2005). RhoA/ROCK signaling regulates Sox9 expression and actin organization during chondrogenesis. Journal of Biological Chemistry 280(12): 11626-11634.

Woods, A., Wang, G., Dupuis, H., Shao, Z. and Beier, F. (2007). Rac1 signaling stimulates N-cadherin expression, mesenchymal condensation, and chondrogenesis. Journal of Biological Chemistry 282(32): 23500-23508.

Yamashiro, T., Wang, X. P., Li, Z., Oya, S., Ã...berg, T., Fukunaga, T., Kamioka, H., Speck, N. A., Takano-Yamamoto, T. and Thesleff, I. (2004). Possible roles of Runx1 and Sox9 in incipient intramembranous ossification. Journal of Bone and Mineral Research 19(10): 1671-1677.

Zanetti, N. C. and Solursh, M. (1984). Induction of chondrogenesis in limb mesenchymal cultures by disruption of the actin cytoskeleton. Journal of Cell Biology 99(1 I): 115-123.

Zeiter, S., Lezuo, P. and Ito, K. (2009). Effect of TGF $\hat{I}^{2} 1$, BMP-2 and hydraulic pressure on chondrogenic differentiation of bovine bone marrow mesenchymal stromal cells. Biorheology 46(1): 45-55.

Zhang, Z., Messana, J., Hwang, N. S. H. and Elisseeff, J. H. (2006). Reorganization of actin filaments enhances chondrogenic differentiation of cells derived from murine embryonic stem cells. Biochemical and Biophysical Research Communications 348(2): 421-427. 